\title{
GMTI PERFORMANCE OF A HIGH RESOLUTION WIDE SWATH SAR OPERATION MODE
}

\author{
Martina Gabele (Martina.Gabele@dlr.de), Gerhard Krieger
}

Microwaves and Radar Institute, German Aerospace Center (DLR)

\begin{abstract}
In this paper an operation mode for space-based SAR/GMTI is suggested which uses a rectangular antenna array and employs subpulse beamsteering on transmit in order to yield wide swath coverage. A signal modeling in rangeDoppler domain is introduced that is suitable for studying the SAR/GMTI performance. Simulation results are given.
\end{abstract}

Index Terms - space-based radar, synthetic aperture radar, ground moving target indication

\section{INTRODUCTION}

Classical synthetic aperture radar (SAR) imaging modes like Stripmap-SAR, Scan-SAR, and Spotlight-SAR require a trade-off between unambiguous swath width and azimuth resolution: Scan-SAR is dedicated to the imaging of wide swaths, however, at an impaired azimuth resolution. SpotlightSAR can be used to improve azimuth resolution at the cost of a lower coverage. Stripmap-SAR has a performance in between Scan-SAR and Spotlight-SAR with respect to coverage and azimuth resolution. The reason for these constraints is that for space-based SAR systems an increase of swath width requires reduction of the pulse repetition frequency (PRF), since a monostatic SAR system cannot transmit and receive at the same time. However, the choice of the PRF is critical, because reduction of PRF raises azimuth ambiguities, while increase of PRF raises range ambiguities.

Next generation space-based SAR/GMTI systems with antenna apertures divided into multiple subapertures with full receiver chains in azimuth and elevation ([1],[2]) will be able to overcome the limitations of classical SAR systems: Beamsteering capability in azimuth and elevation will allow to go beyond the limitations given by the timing diagram, while keeping ambiguities low, thereby enabling wide swath operation modes. One candidate operation mode which is based on the usage of a rectangular array and provides wide swath SAR/GMTI is introduced in the following.

\section{OPERATION MODE}

The wide swath combined SAR/GMTI mode suggested in [2] which we analyze in this paper applies low PRF sampling in order to enable wide swath imaging. A long transmit antenna in azimuth is used in order to transmit large power. Additionally, a narrow transmit antenna pattern keeps azimuth ambiguities low which arise due to the low PRF sampling. The transmit antenna height is small enough in order to provide a beamwidth large enough to illuminate the large swath of interest at once.

Since long integration time is desired, the along-track array has to be split up into multiple subapertures, enabling an integration time limited by the two-way antenna pattern of long transmit antenna and short receive subapertures. Because the splitting into subapertures raises azimuth ambiguities in the subapertures' received signals, it has to be made sure, that the overall along-track array dimension is sufficiently large in order to suppress clutter and azimuth ambiguities in the processing. For elevation beamforming a high receive antenna with multiple subapertures is considered, inducing not only large receiver gain for a wide swath but also providing the possibility to keep range ambiguities low.

Increase of integration time is now achieved by splitting the pulses into multiple subpulses, which are subsequently transmitted to various azimuth directions. If the spacing between the subpulses is narrow, the observable swath between two blind ranges is large, on the other hand range ambiguities are raised.

Increase of swath width is achieved by further splitting the azimuth subpulses into subpulses which are steered to different elevation directions. If the illumination is started at far range and proceeds to near range, the receive window can be shortened. In order to not transmit signal power to blind ranges, the elevation subpulses can be focused such that each elevation subpulse is used for illuminating one observable swath in between two blind range intervals. Further shortening of the receive window can now be achieved by adapting the transmission time spacing between the subpulses such that the echo windows of the elevation subpulses overlap in time, but this again raises range ambiguities caused by the other elevation subpulses. A shorter echo window length allows for longer transmission times and consequently higher average transmit power, but narrows the observable swath width between two blind ranges and increases the width of blind range intervals.

Resolving of blind ranges and blind velocities is possible 
by an appropriate choice of the subpulse azimuth steering angles: Blind ranges can be changed by subpulse beamsteering in azimuth because the traveling time from a range line on a ground swath to the satellite increases with increasing azimuth beamsteering angle off boresight direction. Blind velocities can be changed because the azimuth look angle defines the sensitivity to target motion. This means not only that by use of subpulse beamsteering in azimuth the sensitivity to various directions of target motion can be achieved, but also that blind velocities occuring in the boresight beam can be resolved by use of beams which are steered off boresight.

\section{SIGNAL MODELING}

\subsection{Stationary Target Transfer Function}

Consider a multi-channel SAR system consisting of $N$ channels which are displaced from the array center in along-track direction by $d_{x, n}, n=1, \ldots, N$. The second-order Taylor approximation around $t=0$ of the stationary target range history $R_{n}(t)$ is:

$$
R_{n}(t)=\sqrt{\left(v t-d_{x, n}-x_{0}\right)^{2}+y^{2}} \approx y+\frac{v^{2}}{2 y}(t-\Delta t)^{2},
$$

where $v$ is the platform velocity, $x_{0}$ is the azimuth position of the scatterer, and $\Delta t=d_{x, n} /(2 v)$. We can assume without loss of generality that $x_{0}=0$. Fourier transforming the SAR signal $s_{x, n}(t)=\sqrt{P} \cdot a(t) \cdot \exp \left\{-j 4 \pi R_{n}(t) / \lambda\right\}$ gives [3]:

$$
\begin{aligned}
S_{x, n}(f) & =\sqrt{P} \cdot A_{x}\left(-\frac{y \lambda f}{2 v^{2}}\right) \cdot \exp \left\{-j \frac{\pi d_{x, n}}{v} f\right\} \\
& \cdot \exp \left\{-j \frac{4 \pi y}{\lambda} \sqrt{1-\left(\frac{\lambda f}{2 v^{2}}\right)^{2}}\right\}
\end{aligned}
$$

where $P$ is the received signal power from the scatterer, $a(t)$ is the antenna pattern weighting, and $A_{x}(f)$ is the envelope of its Doppler spectrum. The transfer functions of the along-track array are now stacked to form the steering vector $\mathbf{S}_{N}(f)=\left[S_{x, 1}(f), \ldots, S_{x, N}(f)\right]$.

Splitting each of the $N$ channels into $M$ channels which are displaced from the array phase center in elevation by $d_{z, m}$ and approximating each pulse with a dirac distribution yields the steering vector components $S_{z, m}(\theta), m=1, \ldots, M$, for the across-track array dimension:

$$
S_{z, m}(\theta)=A_{z}\left(\theta-\theta_{t}\right) \cdot \exp \left\{-j \frac{2 \pi d_{z, m}}{\lambda} \sin \left(\theta-\theta_{t}\right)\right\},
$$

where $\theta$ is the target look angle, $\theta_{t}$ the antenna tilt angle, and $A_{z}(\theta)$ the antenna pattern weighting in elevation. In this model we are neglecting any correlation between fast-time samples nor do we consider any range chirp frequency effects. Stacking the transfer functions of the across-track array forms the steering vector $\mathbf{S}_{M}(\theta)=\left[S_{z, 1}(\theta), \ldots, S_{z, M}(\theta)\right]$.
The steering vector $\mathbf{S}(f, \theta)$ of the planar rectangular array is formed by the Kronecker product of along-track and acrosstrack array steering vectors:

$$
\mathbf{S}(f, \theta)=\mathbf{S}_{N}(f) \otimes \mathbf{S}_{M}(\theta) .
$$

\subsection{Moving Target Doppler Spectrum}

Consider now a target moving with constant velocities $v_{x}$ in along-track direction and $v_{y}$ in across-track direction. The second-order Taylor approximation around $t=0$ for the range equation $R_{n}(t)$ becomes:

$$
\begin{aligned}
R_{n}(t) & =\sqrt{\left(\left[v-v_{x}\right] t-d_{x, n}\right)^{2}+\left(y+v_{y} t\right)^{2}} \\
& \approx y+\frac{v_{r e l}^{2}}{2 y}(t-\Delta t)^{2}+v_{y} t
\end{aligned}
$$

with $v_{r e l}=\sqrt{\left(v-v_{x}\right)^{2}+v_{y}^{2}}$, and $\Delta t=d_{x, n}\left(v-v_{x}\right) /\left(2 v_{r e l}^{2}\right)$. The Fourier spectrum $S_{x, n}\left(f, v_{y}\right)$ of the moving target SAR signal can now be found by using the Fourier pair given in equation (3) and applying the Fourier properties of time shift and modulation:

$$
\begin{aligned}
S_{x, n}\left(f, v_{y}\right)= & \sqrt{P_{t}} A_{x}\left(-\frac{y \lambda}{2 v_{r e l}^{2}}[f-\Delta f]\right) \\
& \cdot \exp \left\{-j \frac{4 \pi y}{\lambda} \sqrt{1-\left(\frac{\lambda}{2 v_{r e l}^{2}}[f-\Delta f]\right)^{2}}\right\} \\
& \cdot \exp \left\{-j \frac{\pi d_{x, n}}{v_{e}} f\right\} \exp \left\{j \frac{2 \pi d_{x, n}}{\lambda} \frac{v_{y}}{v_{e}}\right\},
\end{aligned}
$$

with $\Delta f=-2 v_{y} / \lambda$ and $v_{e}=\left(v_{p}-v_{x}\right)+v_{y}^{2} /\left(v_{p}-v_{x}\right)$. The across-track array steering vector $\mathbf{S}_{M}(\theta)$ is assumed the same as for stationary targets.

\subsection{Clutter Covariance Model}

Clutter is the sum of all stationary target signals in a scene. Therefore, as is explained in [5] assuming for one range line infinitesimally narrow clutter reflectivity contributions $d \sigma^{2}$ displaced against each other in slow time by $d t$ the clutter process $c(t)$ can be expressed by a convolution of the SAR impulse response $s(t)$ with the stationary clutter reflectivity process $p(t)$ plus additive white Gaussian noise process $n(t)$. For a system of $N$ channels the cross correlation matrix $\mathbf{r}_{\mathbf{c}}(t)$ characterizing the clutter process as it appears to the multichannel SAR system can be expressed as follows:

$$
\mathbf{r}_{\mathbf{c}}(t)=\mathbf{s}(t) * \mathbf{r}_{\mathbf{p}}(t) * \mathbf{s}^{\dagger}(-t)+\mathbf{r}_{\mathbf{n}}(t),
$$

where $\mathbf{s}(t)=\left[s_{1}(t), s_{2}(t), \ldots, s_{N}(t)\right]$ is the vector of system impulse responses of the $N$ channels, $\mathbf{r}_{\mathbf{p}}(t)$ is the cross correlation matrix of the clutter reflectivity, and $\mathbf{r}_{\mathbf{n}}(t)$ is the cross 
correlation matrix of the additive white Gaussian noise process with:

$$
\mathbf{r}_{\mathbf{n}}(t)=\operatorname{diag}\left(\sigma_{n}^{2} \delta(t), \ldots, \sigma_{n}^{2} \delta(t)\right),
$$

where $\operatorname{diag}(\ldots)$ means diagonal matrix, $\sigma_{n}^{2}$ is the noise power, and $\delta(t)$ denotes the dirac distribution. Assuming a white noise process for the scatterers which means zero correlation between the scatterers on the ground, the cross correlation matrix of the clutter reflectivity process $\mathbf{r}_{\mathbf{p}}(t)$ is:

$$
\mathbf{r}_{\mathbf{p}}(t)=\operatorname{diag}\left(\sigma^{2} \delta(t), \ldots, \sigma^{2} \delta(t)\right) .
$$

By application of the Wiener-Chintschin theorem transformation of random signals into Fourier domain becomes possible. This yields the cross spectral density matrices of the clutter reflectivity $\mathbf{R}_{\mathbf{P}}(f)$ and additive white Gaussian noise $\mathbf{R}_{\mathbf{N}}(f)$ :

$$
\begin{aligned}
& \mathbf{R}_{\mathbf{P}}(f)=\operatorname{diag}\left(\sigma^{2}, \ldots, \sigma^{2}\right), \\
& \mathbf{R}_{\mathbf{N}}(f)=\operatorname{diag}\left(\sigma_{n}^{2}, \ldots, \sigma_{n}^{2}\right) .
\end{aligned}
$$

By use of the Wiener-Lee theorem it can be analyzed how the signal power of an input process is redistributed by a linear time invariant system as is the multi-channel SAR system. This yields the cross spectral density matrix of the clutter process $\mathbf{R}_{\mathbf{C}}(f, \theta)$ as it appears in a multi-channel SAR system:

$$
\begin{aligned}
\mathbf{R}_{\mathbf{C}}(f, \theta) & =\mathbf{S}(f, \theta) \mathbf{R}_{\mathbf{P}}(f) \mathbf{S}^{\dagger}(f, \theta)+\mathbf{R}_{\mathbf{N}}(f) \\
& =\sigma^{2} \mathbf{S}(f, \theta) \mathbf{S}^{\dagger}(f, \theta)+\mathbf{R}_{\mathbf{N}}(f) .
\end{aligned}
$$

\subsection{Azimuth Ambiguities}

In case of low PRF sampling aliasing of the clutter spectrum occurs. Doppler spectra from PRF bands different from the Nyquist band which are folded into the Nyquist band are called azimuth ambiguities. We can express these signals by replacing $f_{k}=f+k P R F, f \in[-P R F / 2, P R F / 2]$, $k=0, \pm 1, \pm 2, \ldots, \pm K$, where $k=0$ is the unambiguous signal. This yields the following expressions for the spectra of the along-track array components:

$$
\begin{aligned}
S_{x, n}\left(f_{k}\right)= & \sqrt{P} \cdot A\left(-\frac{y \lambda f_{k}}{2 v^{2}}\right) \cdot \exp \left\{-j \frac{\pi d_{x, n}}{v} f\right\} \\
& \cdot \exp \left\{-j \frac{4 \pi y}{\lambda} \sqrt{1-\left(\frac{\lambda f}{2 v^{2}}\right)^{2}}\right\} \\
\cdot & \exp \left\{j 2 \pi k t_{0} f\right\} \cdot \exp \left\{j \frac{\pi d_{x, n} P R F}{v} k\right\}
\end{aligned}
$$

where $S_{x, n}\left(f_{0}\right)$ denotes the Nyquist spectrum and $t_{0}=$ $y \lambda P R F /\left(2 v^{2}\right)$. Since different frequencies are pairwise uncorrelated, incorporating azimuth ambiguities yields the following expression for the cross spectral density matrix of the clutter process:

$$
\mathbf{R}_{\mathbf{C}}(f, \theta)=\sigma^{2} \sum_{-K}^{K} \mathbf{S}\left(f_{k}, \theta\right) \mathbf{S}^{\dagger}\left(f_{k}, \theta\right)+\mathbf{R}_{\mathbf{N}}(f) .
$$

\subsection{Range Ambiguities}

Range ambiguities are signals which are received at the same time as the signal of interest, but stem from different transmit pulses. Let us assume that a signal reflected by a scatterer is received at the same time that stems from a pulse which was transmitted $l \Delta T, l=0, \pm 1, \pm 2, \ldots, \pm L$, before/after the pulse from which we expect the received signal from. $l=$ 0 is again the unambiguous signal. The following expressions for the across-track components can be found:

$$
S_{z, m}\left(\theta_{l}\right)=A_{z}\left(\theta_{l}-\theta_{t}\right) \exp \left\{-j \frac{2 \pi d_{z, m}}{\lambda} \sin \left(\theta_{l}-\theta_{t}\right)\right\},
$$

where $\theta_{l}$ is the look angle associated with a slant range $y_{l}=$ $y \pm l \Delta T c / 2$. Since signals from different angles of arrival are pairwise uncorrelated, incorporating range ambiguities yields the following expression for the cross spectral density matrix of the clutter process:

$$
\mathbf{R}_{\mathbf{C}}(f, \theta)=\sigma^{2} \sum_{-L}^{L} \sum_{-K}^{K} \mathbf{S}\left(f_{k}, \theta_{l}\right) \mathbf{S}^{\dagger}\left(f_{k}, \theta_{l}\right)+\mathbf{R}_{\mathbf{N}}(f)
$$

\section{SIMULATION RESULTS}

In the following we exemplarily give some simulation results on the operation mode suggested in section 2 .

\subsection{Eliminating Blind Ranges}

The Timing Diagram indicates whether an echo signal can be received by a system as a function of PRF and look angle, where correct reception means that the echo signal does not interfere with the transmission times of the system. In case of subpulse beamsteering a timing diagram has to be employed for each subpulse. The results from the timing diagrams of the subpulses are then combined in the coverage diagram. This diagram indicates from how many subpulses an echo signal can be received as a function of PRF and look angle. In Figure 1 timing and coverage diagrams are shown for the case of no azimuth beamsteering for the subpulses (a), and in case of azimuth beamsteering for the subpulses (b). In the latter coverage diagram, a swath coverage of e.g. about $10^{\circ}$ look angle interval was achieved for $P R F=2.5 \mathrm{kHz}$.

\subsection{Eliminating Ambiguities}

Ambiguity suppression is performed in a two-step procedure, where first subpulses are extracted by spatial beamforming in elevation, and second clutter and azimuth ambiguities are suppressed by use of Post-Doppler STAP. The signal to interference plus noise ratio loss (SINR loss) is a common measure for comparing adaptive performance. It is defined by the signal to interference plus noise ratio (SINR) divided by the 


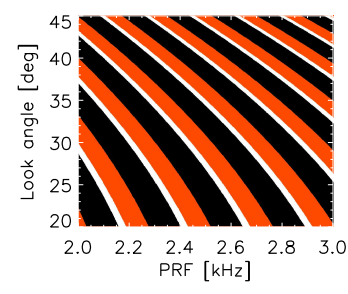

(a) Timing diagram

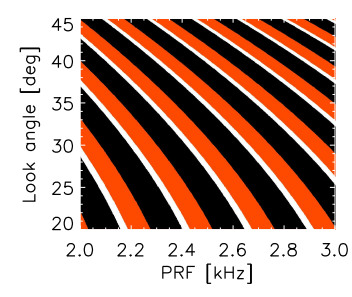

(b) Timing diagram

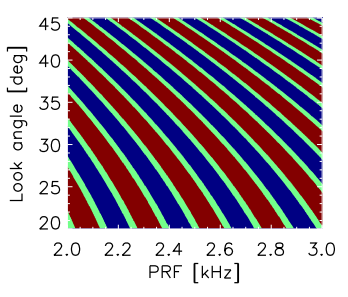

(a) Coverage diagram

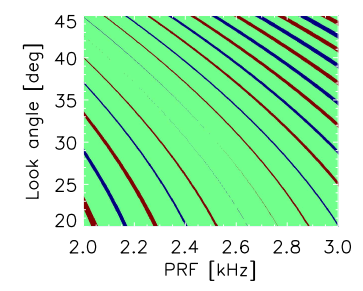

(b) Coverage diagram
Fig. 1. Timing and coverage diagrams without (a) and with (b) subpulse beamsteering in azimuth on transmit (azimuth directions for (b): $\left.\left[0^{\circ}, 14^{\circ}\right]\right)$. Timing diagram: blind range due to transmission of first subpulse (white), second subpulse (orange). Coverage diagram: covered by two subpulses (red), one subpulse (green), no subpulse (blue). System: $576 \mathrm{~km}$ platform height, $7560 \mathrm{~m} / \mathrm{s}$ platform velocity, 0.12 duty cycle, spherical earth model.

interference free signal to noise ratio (SNR) and is a metric between 0 and 1 :

$$
L_{S I N R}\left(f, \theta, v_{y}\right)=\frac{\mathbf{S}^{\dagger}\left(f, \theta, v_{y}\right) \mathbf{R}^{-1}(f, \theta) \mathbf{S}\left(f, \theta, v_{y}\right)}{\mathbf{S}^{\dagger}\left(f, \theta, v_{y}\right) \mathbf{S}\left(f, \theta, v_{y}\right)}
$$

where $\mathbf{S}\left(f, \theta, v_{y}\right)$ is a vector containing the transfer functions of the channels to be combined (elevation or azimuth channels) and $\mathbf{R}^{-1}(f, \theta)$ is the inverse of the corresponding interference plus noise covariance matrix. Low PRF sampling and subpulse beamsteering on transmit place strong requirements on the antenna array. In Figure 2 (a) the SINR loss versus target velocity and PRF is shown. In case of low PRF sampling for the system considered here there are three PRFs for which the system performs well: $1.24 \mathrm{kHz}, 1.54 \mathrm{kHz}, 2.03 \mathrm{kHz}$. The good performance for these PRFs is caused by linear dependencies between the steering vectors of the azimuth ambiguities and depends also on the baselines of the antenna array. In (b) the SINR loss versus antenna height and subpulse spacing is given, if beamforming in elevation is applied in order to decouple the signals of the subpulses before the azimuth processing step. The required antenna height increases strongly in case of narrow subpulse spacing.

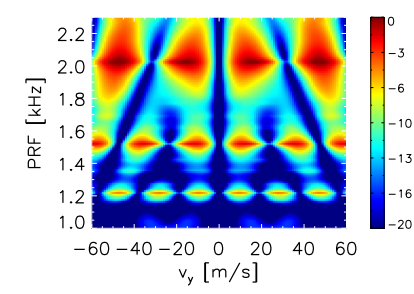

(a) PRF

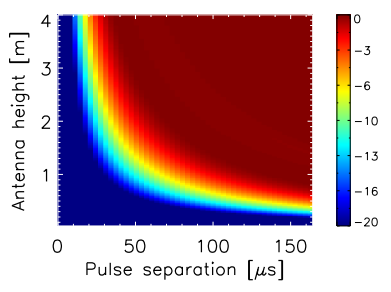

(b) Antenna height
Fig. 2. SINR loss due to PRF and antenna dimensioning. System: $576 \mathrm{~km}$ platform height, $7560 \mathrm{~m} / \mathrm{s}$ platform velocity, $\mathrm{X}$-band system, $14.4 \mathrm{~m}$ antenna length, divided into 6 subapertures for reception. (b): $2.03 \mathrm{kHz} \mathrm{PRF}, 32^{\circ}$ tilt angle, $40^{\circ}$ look angle.

\subsection{Eliminating Blind Velocities}

Various observation directions provide various directions for motion sensitivity. This does not only allow for resolving blind velocity intervals but can also resolve Doppler ambiguities in target motion parameter estimation. For a system as is considered in Figure 2 the clutter notch is about $\Delta v_{y}=$ $\pm 8 \mathrm{~m} / \mathrm{s}$ wide. This implies that in order to resolve a blind velocity for across-track motion at $v_{y}=31 \mathrm{~m} / \mathrm{s}$ as occurs for $P R F=2.03 \mathrm{kHz}$ a steering angle in the order of at least $\phi=45^{\circ}$ is required.

\section{REFERENCES}

[1] Currie, A., Brown, M.A., Wide-swath SAR, IEE Proceedings F - Radar and Signal Processing, vol. 139, pp. 122-135, 1992.

[2] Krieger, G., Gebert, N., Moreira, A., Beamforming Techniques for Spaceborne Radar Remote Sensing, European Conference on Synthetic Aperture Radar (EUSAR), Dresden (Germany), May 2006.

[3] Moreira, A., Mittermayer, J. and Scheiber, R., Extended chirp scaling algorithm for air-and spaceborne SAR data processing in stripmap and ScanSAR imaging modes, IEEE Transactions on Geoscience and Remote Sensing, vol. 34, no. 5, pages 1123-1136, September 1996

[4] Ender, J., Cerutti-Maori, D., W. Bürger, Radar antenna architectures and sampling strategies for space-based moving target recognition, IGARSS, Seoul (South Korea), July 2005.

[5] W.-D. Wirth, Radar techniques using array antennas, Book, ISBN 085296798 5, The Institution of Electrical Engineers, London, United Kingdom, 2001. 